\title{
Endothelial progenitor cells populate the stromal stem niche of tympanum
}

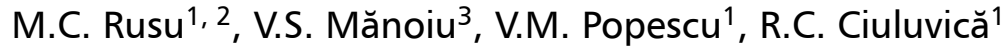 \\ ${ }^{1}$ Division of Anatomy, Faculty of Dental Medicine, "Carol Davila" University of Medicine and Pharmacy, Bucharest, Romania \\ ${ }^{2}$ MEDCENTER - Centre of Excellence in Laboratory Medicine and Pathology, Bucharest, Romania \\ ${ }^{3}$ Department of Cellular and Molecular Biology, National Institute of Research and Development for Biological Sciences, \\ Bucharest, Romania
}

[Received: 21 February 2017; Accepted: 11 April 2017]

The tympanic membrane (TM) integrity is of utmost importance for the sense of hearing. Therefore, the intrinsic potential of the TM to regenerate and repair deserves complete characterisation. Existing studies brought evidence on the epithelial stem niche of the TM. However, the stromal compartment was not evaluated for harbouring a distinctive stem, or progenitor, niche. We aimed doing this in transmission electron microscopy. We used TMs dissected out from 3 male Oryctolagus cuniculus rabbits. Evidence of stromal quiescent stem cells was gathered. Moreover, endothelial progenitor cells were found in the $T M$, being accurately identified by two specific ultrastructural markers of the endothelial lineage: the Weibel-Palade bodies and the stomatal diaphragms of the subplasmalemmal caveolae. The stromal stem niche of the TM appears to be a distinctive contributor during physiological and pathological processes of the TM, such as cholesteatoma formation, at least as a biological support for processes of vasculogenesis. However, further characterisation of the molecular pattern of the stromal stem niche of the TM is mandatory. (Folia Morphol 2017; 76, 4: 630-634)

Key words: ear, caveolae, Weibel-Palade body, endothelial progenitor cells, stem cells

\section{INTRODUCTION}

A common clinical problem worldwide is the tympanic membrane (TM) perforation, which in specific conditions could lead to conductive hearing loss [5].

Current concepts on the mechanisms driving the TM repair involve the epithelial (epidermal) migration $[1,7]$; the peculiar trait of these mechanisms being that the epithelialisation of the soft tissue precedes the advancement of the fibrous tissue [2].
Two different migratory waves, slow and fast, seem to distinctively involve the cells of the stratum basale and those of the stratum corneum, respectively [1]. Nevertheless, the epithelial stem niches of tympanum are indicated as being the umbo, the annular region and along the malleus [6].

We took into account the concept of the universal perivascular/periendothelial stem niche [9] which relies upon the recruitment of pericytes as

Address for correspondence: Dr. M.C. Rusu, Division of Anatomy, Faculty of Dental Medicine, "Carol Davila" University of Medicine and Pharmacy, 8 Bd. Eroilor Sanitari, RO-050474, Bucharest, Romania, tel: +40722363705, fax: +40213212284, e-mail: anatomon@gmail.com

All authors have equally contributed to this paper. 
mesenchymal stem (stromal) cells (MSCs) within the connective stroma [16]. We therefore hypothesized that the lamina propria could qualify as the stromal stem niche of the TM and we aimed thus at identifying in transmission electron microscopy (TEM) whether, or not, it harbours undifferentiated stem and/or progenitor cells.

\section{MATERIALS AND METHODS}

In this study were included 3 male Oryctolagus cuniculus rabbits weighing $2.5-3 \mathrm{~kg}$, aged between 6 and 12 months. Experimental animals were housed in individual cages with fed ad libitum and 12/12-h light/dark cycle. All rabbits were acclimatised for 1 week before the experiments started. After 8 days animals were killed with lethal intravenous injections of phenobarbitone. All procedures were approved by the Institutional Bioethics Committee. The tympanic membranes were dissected out and further processed for TEM. Small tissue fragments about $1-2 \mathrm{~mm}^{3}$ were prefixed in fresh ice-cold $4 \%$ glutaraldehyde in sodium cacodylate buffer, $\mathrm{pH} 7.4$ for $4 \mathrm{~h}$ at $4^{\circ} \mathrm{C}$. After fixation, the tissues were $6^{\mathrm{x}}$ washed in $0.05 \mathrm{M}$ sodium cacodylate buffer ( $\mathrm{pH} 7.4)$ at $4^{\circ} \mathrm{C}$, postfixed in $2 \%$ osmium tetroxide in $0.1 \mathrm{M}$ sodium cacodylate at room temperature for $2.5 \mathrm{~h}$, stained en bloc with $0.5 \%$ aqueous uranyl acetate overnight at $4^{\circ} \mathrm{C}$ and washed with $0.05 \mathrm{M}$ sodium cacodylate buffer. After dehydration in graded series of ethanol and infiltration with propylene oxide, specimens were embedded in Glycid ether (Epon 812-equivalent) and were finally polymerised at $60^{\circ} \mathrm{C}$ for $48 \mathrm{~h}$. Semithin sections were stained with $1 \%$ toluidine blue for light microscopy. Ultrathin sections $(80-100 \mathrm{~nm})$ were cut using a diamond knife and collected on 200 mesh copper grids, and double counterstained with uranyl acetate and subsequently lead citrate. The grids were examined with a Philips electron microscope EM 208S operated at an acceleration voltage of $80 \mathrm{kV}$. We used an image acquisition system consisting of a video camera Veleta and the iTEM Olympus Soft Imaging System.

\section{RESULTS}

On grids there were accurately identified the epidermal layer (with its layers: stratum corneum, stratum granulosum, stratum spinosum and stratum basale) and the lamina propria of the tympanum. Within the basal layer of the outer epithelium of tympanum were identified cells in various nuclear stages, pre-mitotic or post-mitotic (Fig. 1).

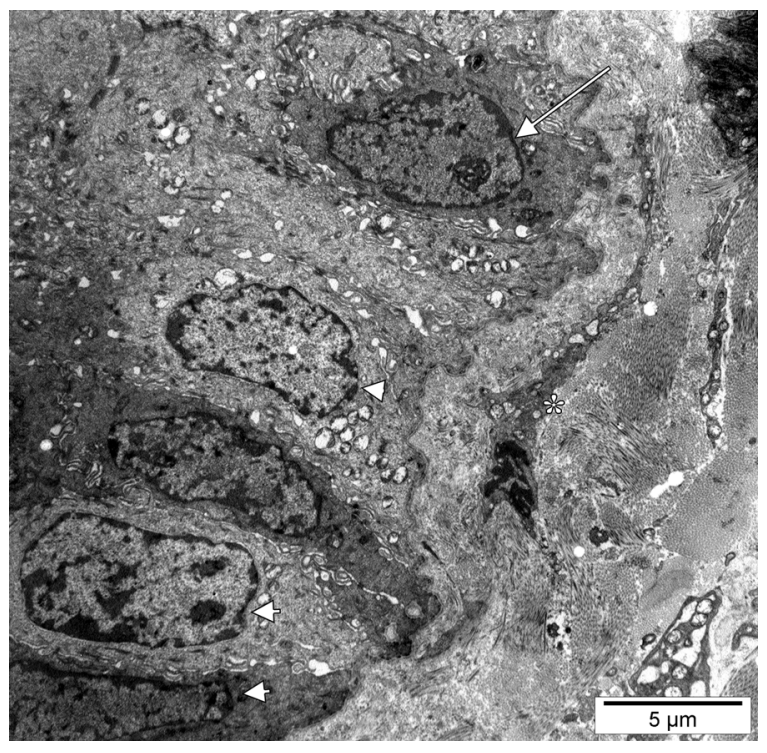

Figure 1. Rabbit tympanum. Transmission electron microscopy. Within the basal epidermal layer of tympanum the cells display either nuclei with pronounced nucleolus (arrow) or nuclei with barely undetectable nucleoli or open DNA (arrowheads). A subepidermal collagen-embedded fibroblastoid cell $\left({ }^{*}\right)$ is indicated.

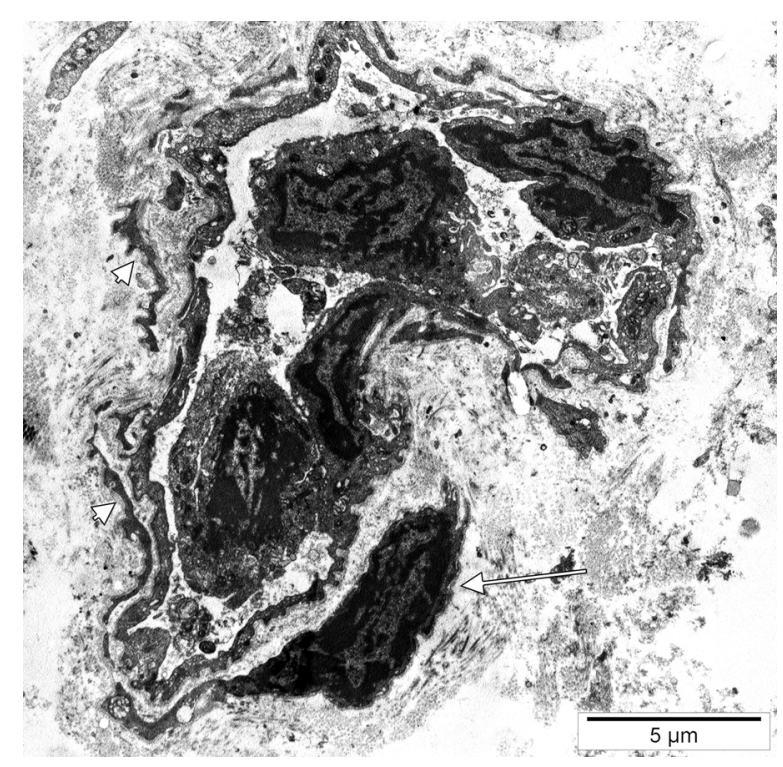

Figure 2. Rabbit tympanum. Transmission electron microscopy. A pericyte cell body with large nuclear/cytoplasmic $(\mathrm{N} / \mathrm{C})$ ratio (arrow) and pericyte cell processes (arrowheads) are identified in a microvessel of tympanum.

Within the lamina propria of tympanum microvessels were present. Their walls consisted of endothelial cells covered by pericytes embedded within the endothelial basal lamina (Figs. 2, 3). 


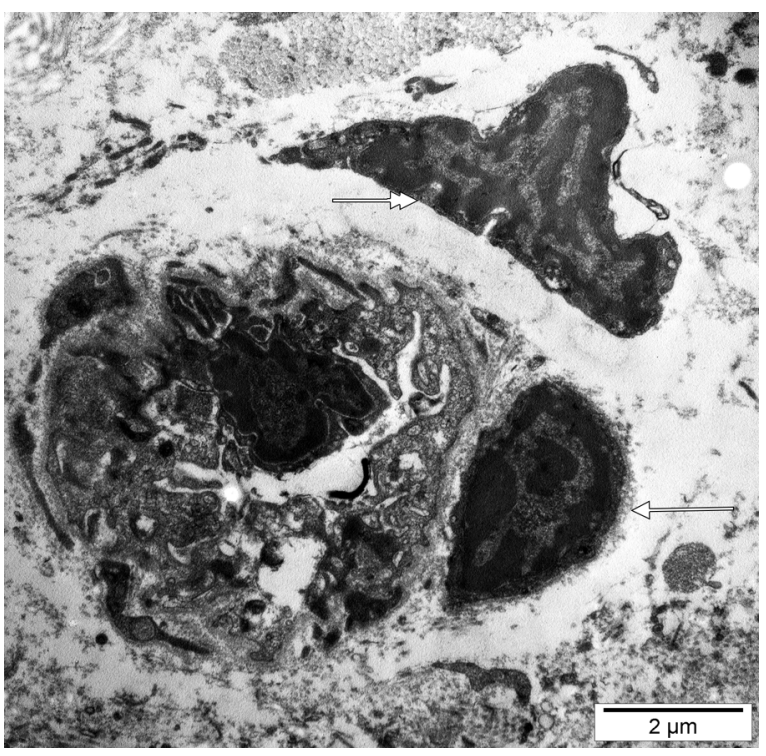

Figure 3. Rabbit tympanum. Transmission electron microscopy. Periendothelial (arrow) and perivascular (double-headed arrow) cells with stem ultrastructural phenotypes are identified.

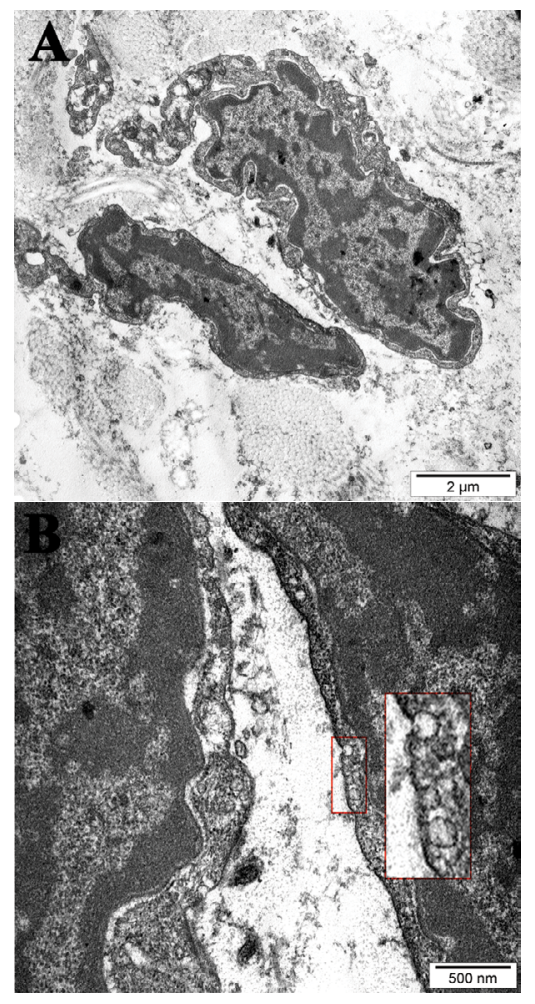

Figure 4. Rabbit tympanum. Transmission electron microscopy. Stromal cells with large nuclear/cytoplasmic $(\mathrm{N} / \mathrm{C})$ ratio $(\mathbf{A}$, general view) depict scarce caveolae obturated by stomatal diaphragms (B, inset - magnified detail).

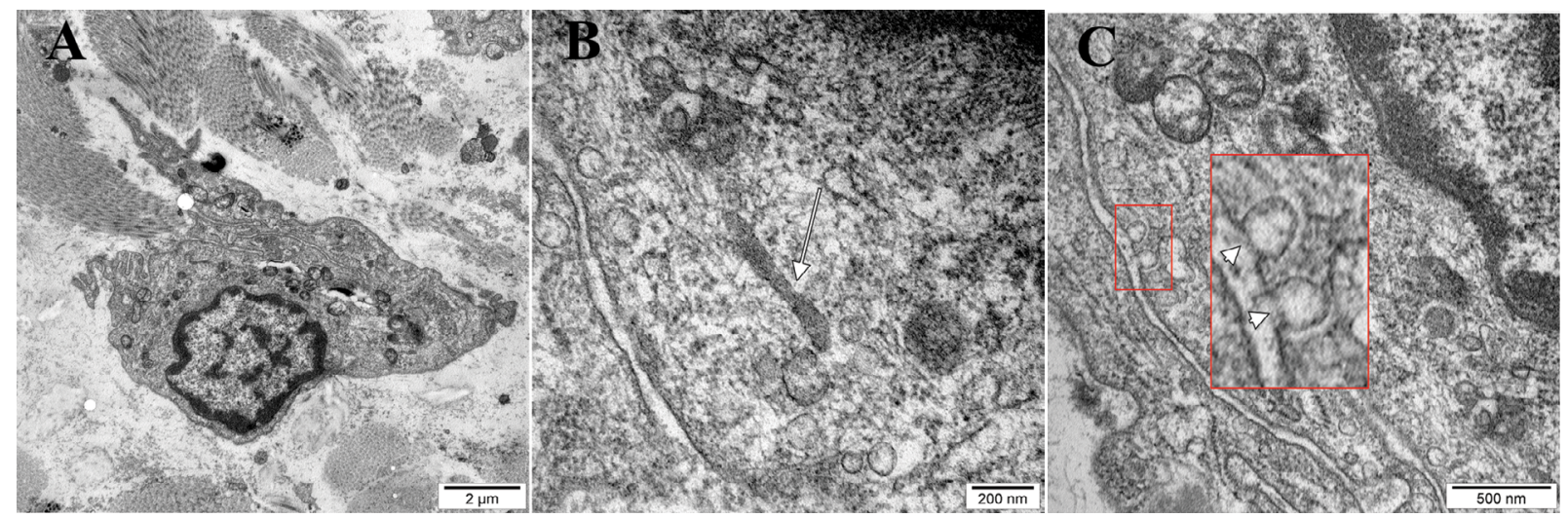

Figure 5. Rabbit tympanum. Transmission electron microscopy; A. General aspect of a stromal but not vascular mural cell; B. Evidence of a Weibel-Palade body (arrow) indicates an endothelial progenitor phenotype of that cell; C. Stomatal diaphragms (inset, magnified detail, arrowheads) obturate the plasmalemmal caveolae of the endothelial progenitor cell.

Interestingly, the cell body of such "dark" pericytes had a large nuclear/cytoplasmic (N/C) ratio, their nuclei being heterochromatic, with marginal and clustered condensed chromatin; few organelles filled the poorly-represented cytoplasm of these cells. This ultrastructural phenotype was assumed being a quiescent stem-like one. Moreover, perivascular dark cells with similar phenotypes were assessed (Fig. 3), being not embedded within the basal endothelial lamina.

Isolated or clustered stromal cells with stem-like phenotypes were found also embedded in collagen 


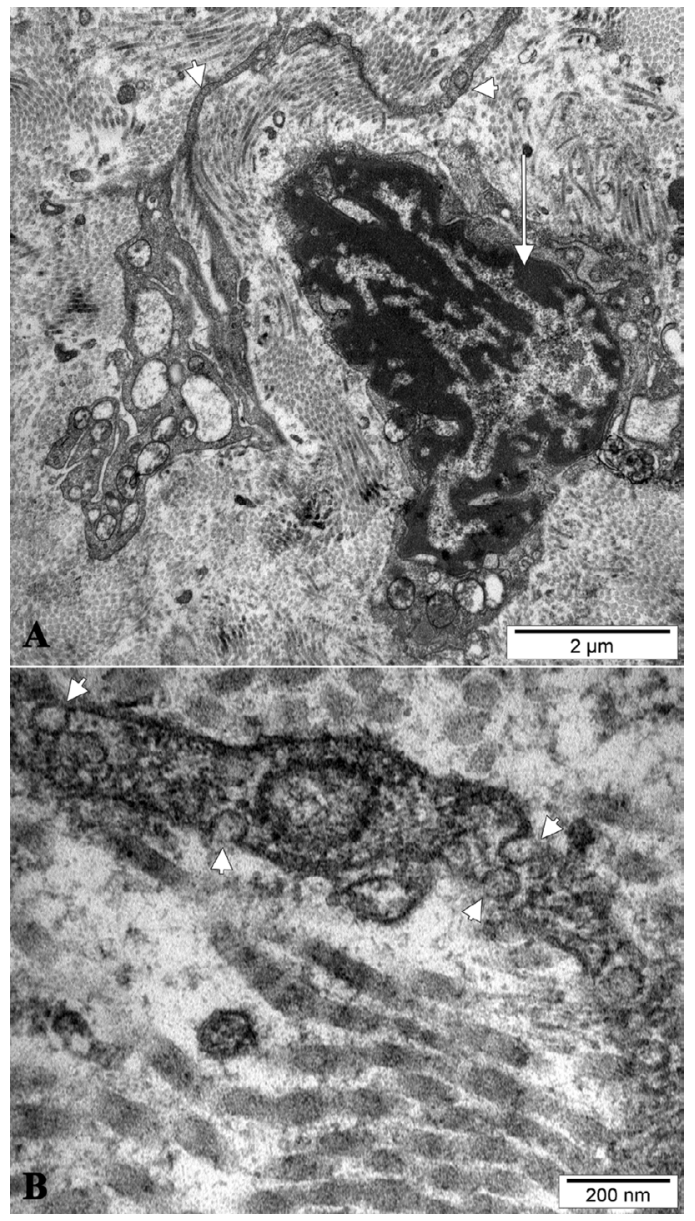

Figure 6. Rabbit tympanum. Transmission electron microscopy. A stromal cell with large nuclear/cytoplasmic $(\mathrm{N} / \mathrm{C})$ ratio, heterochromatic and indented nucleus ( $\mathbf{A}$, arrow) neighbours thin telopodial prolongations of stromal cells (A, arrowheads) which display subplasmalemmal caveolae obturated by stomatal diaphragms (B, arrowheads).

and not related anatomically to microvessels. There were frequent instances when such dark quiescent stem cells were building tandems with neighbour cells with a lighter appearance but also displaying an undifferentiated pattern (Fig. 4). Such dark and light stem, or progenitor, cells scarcely presented plasmalemmal specialisations, such as clathrincoated vesicles and/or subplasmalemmal caveolae which were closed/obturated by stomatal diaphragms (Figs. 4-6). We also found Weibel-Palade bodies in the lighter type of stem/progenitor cells' cytoplasm. So, these un-, or poor-differentiated cells were assessed as belonging to the endothelial lineage, thus being endothelial progenitor cells (EPCs). It is also relevant for the discussion to note here that stromal processes resembling telopodes, the processes of telocytes, also presented caveolae obturated by stomatal diaphragms (Fig. 6).

\section{DISCUSSION}

It has been widely accepted that most acute TM perforations heal spontaneously [5]. However, most of the studies dealing with the stem niche of TM are focused on its epidermal, epithelial, component. We demonstrated here that bona fide stem and/or progenitor cells are putative residents of the fibrous layer of the TM, thus being potential players during physiological and pathological TM regeneration and repair.

We found however discrete differences of phenotype indicating that different subsets of stem and progenitor cells harbour the stromal stem niche of the TM. We found dark quiescent stem cells with poorly represented cytoplasm and organelles, as well as incompletely differentiated cells which had a lighter appearance in TEM. This fulfils the stem niche definition in which committed progenitor cells join the niche and provide feedback to their stem parents [3]. Nevertheless, finding periendothelial, as well as perivascular stem (stromal) mesenchymal cells demonstrates that the concept of the universal perivascular stem niche convincingly applies also to the TM. In this regard, TM damage repair equally and actively involves the epithelium and the stromal compartment of the TM in which processes of stem cell differentiation and neovessel formation occur. This is indirectly supported by studies on the middle ear cholesteatoma during which proliferating tissues require an enhanced blood supply: angiogenesis is a prerequisite for the expansion of cholesteatoma which is supported by rapidly growing keratinocytes as well as stromal cells [14]. So, although the epithelial stem niche could be a first player during TM perforation repair, the stromal niche is equipped, as we found, with cells able to support the expansion of cholesteatoma.

As we found, the stromal niche of the TM embeds EPCs which were firmly identified in TEM by two distinctive morphological features: the Weibel-Palade bodies and the obturated subplasmalemmal caveolae. The Weibel-Palade bodies are endothelial-specific organelles which house von Willebrand factor, being thus clearly related to the coagulation system [15]. On the other hand, the stomatal diaphragms of caveolae, which are thin protein barriers obturating the openings of the subplasmalemmal caveolae (or plasmalemmal vesicles), are also indicative for cells of the endothelial 
lineage $[8,11-13]$. Finding such stomatal diaphragms of caveolae in telopodial prolongations of TM stromal cells is also indicative for an endothelial progenitor phenotype. This is because arguments were brought for a subset of telocytes being in fact endothelial progenitors [10]. However, although mesenchymal stem cells and endothelial progenitors could be supplied by the perivascular niches, a bone marrow supply of such cells could not be discarded.

We did not find any study indicating, as we did here, that neovessel formation in the TM could be supported by EPCs able to support local processes of adult vasculogenesis. In turn, the expression of the vascular endothelial growth factor (VEGF) receptor is indicative for the beginning of the processes of vasculogenesis and it was proven that VEGF is upregulated in tympanomastoid cholesterol granulomas [4]. It is therefore reasonable to speculate that the resident EPCs of the TM could have a direct involvement during the processes of neovessel formation in the TM. Moreover, although viewed as a unique mechanism of neovessel formation within the TM, angiogenesis enables and support the migration of keratinocytes [14]; thus, the stromal niche of the TM which is equipped with endothelial progenitors is able to support the epithelium-driven repair of the TM perforations.

\section{CONCLUSIONS}

The stromal stem niche of tympanum adds so to the epithelial one to complete the anatomy of the TM stem niche. Further immunohistochemical studies are mandatory to characterise the stromal stem niche of tympanum.

\section{REFERENCES}

1. Boedts D. Tympanic epithelial migration. Clin Otolaryngol. 1978; 3(3): 249-253, doi: 10.1111/j.13652273.1978.tb00695.x.

2. Gladstone HB, Jackler RK, Varav K. Tympanic membrane wound healing. An overview. Otolaryngol Clin North Am. 1995; 28(5): 913-932, indexed in Pubmed: 8559580.

3. Hsu YC, Fuchs E. A family business: stem cell progeny join the niche to regulate homeostasis. Nat Rev Mol
Cell Biol. 2012; 13(2): 103-114, doi: 10.1038/nrm3272, indexed in Pubmed: 22266760.

4. Iannella G, Di Gioia C, Carletti R, et al. Tympanomastoid cholesterol granulomas: Immunohistochemical evaluation of angiogenesis. Laryngoscope. 2017; 127(8): E283-E290, doi: 10.1002/lary.26458, indexed in Pubmed: 28158903.

5. Kim SW, Kim J, Seonwoo H, et al. Latent progenitor cells as potential regulators for tympanic membrane regeneration. Sci Rep. 2015; 5: 11542, doi: 10.1038/ srep11542, indexed in Pubmed: 26100219.

6. Knutsson J, von Unge M, Rask-Andersen H. Localization of progenitor/stem cells in the human tympanic membrane. Audiol Neurootol. 2011; 16(4): 263-269, doi: 10.1159/000320612, indexed in Pubmed: 21051884.

7. Makino K, Amatsu M, Kinishi M, et al. Epithelial migration in the healing process of tympanic membrane perforations. Eur Arch Otorhinolaryngol. 1990; 247(6): 352-355, indexed in Pubmed: 2278700.

8. Palade GE, Bruns RR. Structural modulations of plasmalemmal vesicles. J Cell Biol. 1968; 37(3): 633-649, indexed in Pubmed: 11905197.

9. Perlea P, Rusu MC, Didilescu AC, et al. Phenotype heterogeneity in dental pulp stem niches. Rom J Morphol Embryol. 2016; 57(4): 1187-1193, indexed in Pubmed: 28174783.

10. Rusu MC, Hostiuc S, Vrapciu AD, et al. Subsets of telocytes: Myocardial telocytes. Ann Anat. 2017; 209: 37-44, doi: 10.1016/j.aanat.2016.09.006, indexed in Pubmed: 27777113.

11. Stan RV. Structure of caveolae. Biochim Biophys Acta. 2005; 1746(3): 334-348, doi: 10.1016/j.bbamcr.2005.08.008, indexed in Pubmed: 16214243.

12. Stan RV. Endothelial stomatal and fenestral diaphragms in normal vessels and angiogenesis. J Cell Mol Med. 2007; 11(4): 621-643, doi: 10.1111/j.15824934.2007.00075.x, indexed in Pubmed: 17760829.

13. Stan RV, Tkachenko E, Niesman IR. PV1 is a key structural component for the formation of the stomatal and fenestral diaphragms. Mol Biol Cell. 2004; 15(8): 3615-3630, doi: 10.1091/mbc.E03-08-0593, indexed in Pubmed: 15155804.

14. Sudhoff H, Dazert S, Gonzales AM, et al. Angiogenesis and angiogenic growth factors in middle ear cholesteatoma. Am J Otol. 2000; 21(6): 793-798, indexed in Pubmed: 11078065.

15. Weibel ER. Fifty years of Weibel-Palade bodies: the discovery and early history of an enigmatic organelle of endothelial cells. J Thromb Haemost. 2012; 10(6): 979-984, doi: 10.1111/j.1538-7836.2012.04718.x, indexed in Pubmed: 22646831

16. Zimmerlin L, Donnenberg VS, Donnenberg AD. Pericytes: a universal adult tissue stem cell? Cytometry A. 2012; 81(1): 12-14, doi: 10.1002/cyto.a.21168, indexed in Pubmed: 22069300. 